\title{
Zweiter PCSK9-Hemmer zur starken LDL-Senkung
}

— Seit November 2015 ist mit Alirocumab $\left(\right.$ Praluent ${ }^{\circledR}$ ) der zweite PCSK9-Inhibitor zur potenten Cholesterinsenkung verfügbar. Zwei Dosierungsstufen ermöglichen eine Stufentherapie. Eingesetzt wird der alle 14 Tage s.c. zu injizierende monoklonale Antikörper bei Hochrisikopatienten mit primärer Hypercholesterinämie und hohen Cholesterinwerten trotz - und das ist wichtig ausgereizter herkömmlicher lipidsenkender Therapie.

Wie Prof. Christopher Cannon, Boston, erklärte, reduzieren die beiden zugelassenen PCSK9-Hemmer Alirocumab und Evolocumab das LDL-Cholesterin zuverlässig und konsistent um mehr als 50\% - unabhängig von einer Statineinnahme oder einer familiären Hypercholesterinämie. Vorläufige Daten deuten zudem an, dass die Therapie kardiovaskuläre Komplikationen verhindern könnte. Ergebnisse aus drei großen Studien werden in zwei Jahren erwartet.
Die Antikörpertherapie werde in der Regel gut vertragen - auch dann, wenn Studienpatienten sehr tiefe LDL-Cholesterinwerte von unter $25 \mathrm{mg} / \mathrm{dl}$ erreichten. Bisher, so Cannon, gibt es keine Hinweise auf schwerwiegende Nebenwirkungen.

\section{Zwei Dosierungsstufen}

Alirocumab kommt in zwei Dosierungen auf den Markt, einer Startdosis von $75 \mathrm{mg} / \mathrm{d}$ und einer 150-mg/d-Dosis, auf die bei Bedarf erhöht werden kann. Nach dieser Stufenstrategie wurde im klinischen Studienprogramm ODYSSEY in der Regel verfahren.

Eine beim AHA-Kongress präsentierte Post-hoc-Analyse gepoolter Daten von sechs Studien mit 1.291 Patienten mit Hypercholesterinämie zeigte, dass bei $74 \%$ der Patienten $75 \mathrm{mg} / \mathrm{d}$ genügten. Sie kamen innerhalb von acht Wochen auf ihren LDL-Zielwert, der je nach Risikokonstellation bei $<70 \mathrm{mg} / \mathrm{dl}$ oder

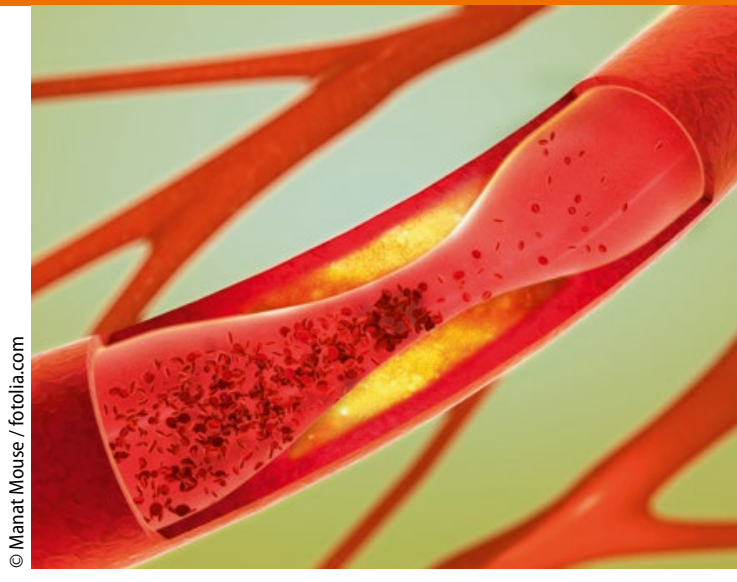

Schlechte Chancen für den Pfropf.

$<100 \mathrm{mg} / \mathrm{dl}$ lag. Beim verbleibenden Viertel der Patienten wurde die Dosis nach 12 Wochen verdoppelt. Dadurch sank das LDL-Cholesterin nochmals um $14,2 \%$, weitere $61 \%$ dieser Patienten erreichten ihre Zielwerte, berichtete Dr. Harold Bays, Kentucky. Zum Vergleich: Eine Dosisverdopplung bei Statinen führt im Schnitt nur zu einer zusätzlichen LDL-Senkung um ca. 6\%.

Dr. Dirk Einecke

- Poster Sessions; CME-Symposium „New Frontiers and Emerging Treatments Paradigms for Optimizating LDL-Targeted Cardiovascular Risk Reduction", American Heart Association Scientific Sessions 2015; Orlando/USA, 7.-11. November 2015 (unterstützt von Sanofi und Regeneron)

\section{Chronische Urtikaria}

\section{Umfrage belegt hohe Alltagsbelastung durch juckende Quaddeln}

_ Die Online-Umfrage ATTENTUS dokumentiert erstmals direkt die Alltagsbelastung von Patienten mit diagnostizierter chronischer Urtikaria. Über 9.000 äußerten sich zur Krankheitslast, dem Einfluss auf den Alltag, zu ihren Arztbesuchen sowie dem Effekt von Medikamenten [Br J Dermatol 2016, online 4. Januar. doi: $10.1111 /$ bjd.14203].

Anhand einer Punkteskala von 1 (nicht beeinträchtigt) bis 6 (extrem beeinträchtigt) konnten die Betroffenen ihre Alltagsbelastung angeben. Sie waren mehrheitlich weiblich $(80,4 \%)$ und im Mittel 26,7 Jahre alt. Ein Drittel gab an, im Alltag beeinträchtigt bis stark beeinträchtigt zu sein. $38,1 \%$ dieser Patienten litten über das gesamte Jahr hinweg an Urtikaria-Symptomen.

\section{Nur $40 \%$ sind in Behandlung}

Bedenklich erscheint, dass nur $40 \%$ der Patienten mit Symptomen überhaupt in ärztlicher Betreuung waren. Besonders Patienten, die schon 15 Jahre oder länger erkrankt waren, blieben den Praxen fern. 53,8\% glaubten nicht mehr daran, dass ein Arzt ihnen helfen könne, während $42,9 \%$ überzeugt waren, die Symptome selbst am besten behandeln zu können.
In diesem Zusammenhang verweisen die Autoren auf die aktuelle internationale EAACI/WAO-Leitlinie, die das Therapieziel „völlige Beschwerdefreiheit” empfehle. Auf der ersten Behandlungsstufe der Leitlinie werden standardmäBig nicht-sedierende $\mathrm{H}_{1}$-Antihistaminika eingesetzt, die jedoch bei vielen Patienten nicht ausreichend helfen [World Allergy Organ J. 2012;5:125-147]. Hier kommen dann Mittel wie der monoklonale Antikörper Omalizumab (Xolair) zum Einsatz.

Red.

- Nach Informationen von Novartis 